\title{
Suboptimal Transmission of Orthogonal Space-Time Block Codes Over Correlated Distributed Antennas
}

\author{
Shuangfeng Han, Member, IEEE, Shidong Zhou, Member, IEEE, Jing Wang, Senior Member, IEEE, \\ Victor O. K. Li, Fellow, IEEE, and Kyung Park, Member, IEEE
}

\begin{abstract}
This letter investigates optimal transmission of orthogonal space-time block codes (OSTBCs) over distributed antennas (DAs) in non-ergodic flat Rayleigh fading channels with transmit antenna correlations. A generalized DA topology is considered, where the DAs are grouped into some geographically dispersed ports within each of which the DAs are co-located. Assuming equal power allocation within each port, the outage probability is derived. We find that minimizing the outage probability only requires the feedback of the eigenvalues of the transmit correlation matrix at the transmitter. Since it is computationally intensive to minimize the outage probability, an antenna subset selection with suboptimal power allocation scheme is proposed, whose effectiveness has been demonstrated by numerical results.
\end{abstract}

Index Terms-Distributed antennas (DAs), orthogonal space-time block codes (OSTBCs), outage probability.

\section{INTRODUCTION}

I NFORMATION theoretic results in [1] and [3] have demonstrated the capacity advantage of distributed antennas (DAs) over point-to-point multiple-input multiple-output (MIMO) channels because of the inherent macroscopic diversity and shortened average access distance. Recently, there have been growing interests in combining space-time codes with DAs. For example, spatial multiplexing schemes are developed in [7] to achieve a high spectral efficiency. When high diversity gain is required, orthogonal space-time block codes (OSTBCs) [5] can be utilized due to their decoding simplicity, e.g., the work in [8].

We focus in this letter on applying OSTBCs over DAs. Without channel state information (CSI) at the transmitter, equal power transmission of OSTBCs over geographically dispersed DAs may result in unacceptable performance because

Manuscript received March 3, 2006; revised June 5, 2006. This work was supported in part by the National Natural Science Foundation of China under Grant 90204001 and in part by ETRI of Korea. The associate editor coordinating the review of this manuscript and approving it for publication was Prof. Zhengdao Wang.

S. Han was with the Electronics Engineering Department, Tsinghua University, Beijing, China, and is now with Samsung Electronics, Suwon, Korea (e-mail: shuangf.han@samsung.com).

S. Zhou and J. Wang are with the Electronics Engineering Department, Tsinghua University, Beijing, China (e-mail: zhousd@ @singhua.edu.cn; wangj@tsinghua.edu.cn).

V. O. K. Li is with the Department of Electrical and Electronic Engineering, University of Hong Kong, Pokfulam, Hong Kong, China (e-mail: vli@eee.hku. hk).

K. Park was with the Electronics and Telecommunications Research Institute of Korea and is now with the Mobile Communication Standardization Team, PANTECH Co., Seoul, Korea (e-mail: park.kyung@pantech.com).

Digital Object Identifier 10.1109/LSP.2006.882096

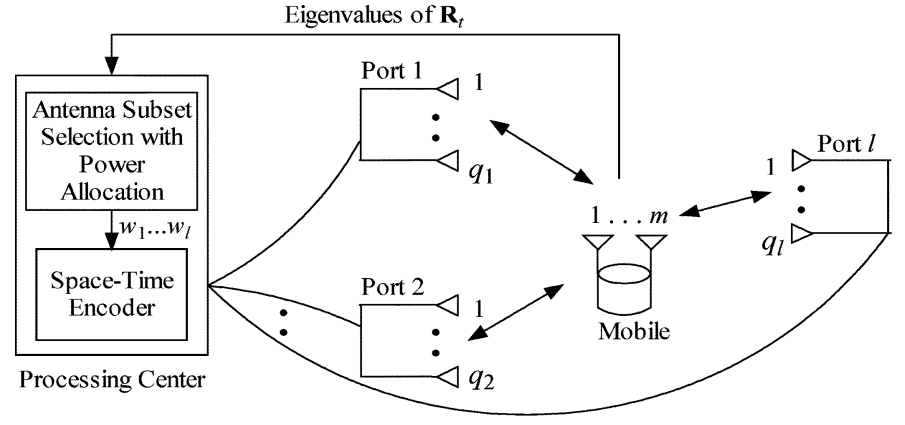

Fig. 1. $\left(n, l, q_{1}, \ldots, q_{l}, m\right)$ DA topology.

the DAs with severe large-scale fading may waste much power. Therefore, transmit power allocation to the DAs based on some CSI feedback is indispensable. Prior work in [8] has studied the outage probability of OSTBCs in Nakagami fading channels, but the optimal power allocation problem has not been solved. To tackle this problem, we consider a generalized DA topology, i.e., the DAs are grouped into some geographically dispersed ports where the DAs are co-located. This topology seems more practical since deploying fully scattered DAs is generally rather difficult in some scenarios. For simplicity of system implementation, we assume equal power allocation within each port and explore how to optimally allocate transmit power to different ports to minimize the outage probability in non-ergodic flat Rayleigh fading channels with transmit antenna correlations. The contribution of this letter includes the derivation of the outage probability in closed forms and an antenna subset selection with suboptimal power allocation scheme whose performance approaches optimal.

\section{SYSTEM MODEL}

Consider a single user $\left(n, l, q_{1}, \ldots, q_{l}, m\right)$ DA topology. As shown in Fig. 1, $n$ DAs are grouped into $l$ geographically dispersed ports with $q_{j}$ co-located DAs in the $j$ th port, with $\sum_{j=1}^{l} q_{j}=n$, and $m$ mobile antennas are co-located. All the DAs have independent connections to the processing center that controls the transmit and receive signals of each DA. One example of this mechanism can be found in the distributed wireless communication system [4]. Generally, the macroscopic and microscopic fading experienced at different antenna ports are independent. However, transmit correlations are often observed within each port due to the DA placement or the geometry in the transmission scenarios. We focus on the analysis of transmit correlations and assume the receive antennas are uncorrelated. 
At the processing center, OSTBCs' symbols with unit average power are generated by the space-time encoder and are multiplied by the power allocation matrix $\mathbf{P}$ before transmission. We assume the channel is non-ergodic and frequency flat. The received signal can be written as

$$
\mathbf{y}=\sqrt{P} \mathbf{H P}^{1 / 2} \mathbf{x}+\mathbf{n}=\sqrt{P} \mathbf{H}_{w} \mathbf{R}_{t}^{1 / 2} \mathbf{P}^{1 / 2} \mathbf{x}+\mathbf{n}
$$

where $\mathbf{y}$ and $\mathbf{x}$ are receive and transmit vectors, $\mathbf{n}$ is an $m \times$ 1 noise vector with independent identically distributed (i.i.d.) complex $N\left(0, \sigma^{2}\right)$ entries, and $P$ is the total average transmit power. $P / \sigma^{2}$ is the transmit power to receive noise ratio (TSNR) and is denoted by $\rho$. The composite channel matrix $\mathbf{H}$ is expressed as $\mathbf{H}_{w} \mathbf{R}_{t}^{1 / 2}$ [6], where $\mathbf{H}_{w}$ is an $m \times n$ matrix with i.i.d. complex $N(0,1)$ entries, and $\mathbf{R}_{t}=\mathrm{E}\left(\mathbf{H}^{\mathrm{H}} \mathbf{H}\right) / m$ is the $n \times n$ transmit correlation matrix with $\mathrm{E}\{$.$\} and superscript \mathrm{H}$ denoting the expectation and Hermitian transpose of a matrix, respectively. Actually, $\mathbf{R}_{t}=\operatorname{diag}\left(a_{1} \mathbf{R}_{t, 1}, \ldots, a_{l} \mathbf{R}_{t, l}\right)$, where $\mathbf{R}_{t, j}, j=1, \ldots, l$ denotes the $q_{j} \times q_{j}$ normalized (with diagonal entries of 1) transmit correlation matrix of the $j$ th port, which has $t_{j}, 1 \leq t_{j} \leq q_{j}$ positive eigenvalues $\lambda_{j, k}, k=$ $1, \ldots, t_{j}$, and $a_{j}$ represents the corresponding large-scale fading (shadow fading and path loss). Note that $a_{j} \lambda_{j, k}, j=1, \ldots, l$, $k=1, \ldots, t_{j}$ are eigenvalues of $\mathbf{R}_{t}$, and we assume they are known at the transmitter through feedback. At the receiver, accurate CSI is assumed. Assuming equal power allocation within each port, the diagonal power allocation matrix $\mathbf{P}$ can be given as $\mathbf{P}=\operatorname{diag}\left(w_{1} / q_{1}, \ldots, w_{1} / q_{1}, \ldots, w_{l} / q_{l}, \ldots, w_{l} / q_{l}\right)$, where $w_{j}, j=1, \ldots, l$ denotes the power allocation weight to the $j$ th port, with $\sum_{j=1}^{l} w_{j}=1$. We define transmit antenna subset as any combination of DA ports and denote the possible $2^{l}-1$ subsets by $\mathrm{A}_{1}, \ldots, \mathrm{A}_{2^{l}-1}$. For OSTBCs' transmission over subset $\mathrm{A}_{k}\left(k=1, \ldots, 2^{l}-1\right)$, we assume only one rate $r_{k}$.

Outage capacity is a useful characterization for non-ergodic fading channels where channel matrix $\mathbf{H}$ is random but held constant for each use of the channel. For a given data rate $R$ and TSNR level $\rho$, our target is to optimally select the antenna subset and allocate power to minimize the outage probability.

\section{DERIVATION OF THE OUTAGE PROBABILITY}

Following the procedure outlined in [2], for OSTBCs transmitted over subset $\mathrm{A}_{l}$ (assumed to include all $l$ ports) with rate $r_{l}$ and positive power allocation weights $w_{1}, \ldots, w_{l}$, the normalized capacity for a given channel realization can be expressed as

$$
C=r_{l} \log _{2}(1+\eta)=r_{l} \log _{2}\left(1+\left\|\mathbf{H}_{w} \mathbf{R}_{t}^{1 / 2} \mathbf{P}^{1 / 2}\right\|_{F}^{2} \rho\right)
$$

where $\|\cdot\|_{F}$ denotes the Frobenius norm, and $\eta$ is the SNR at detection, which can be further derived as

$$
\eta=\sum_{j=1}^{l} a_{j} w_{j} \rho / q_{j} \sum_{k=1}^{t_{j}} \lambda_{j, k} \sum_{z=1}^{m}\left|h_{z}\right|^{2}=\sum_{j=1}^{l} \sum_{k=1}^{t_{j}} \eta_{j, k}
$$

with i.i.d. complex $N(0,1)$ random variables $h_{z}, z=1, \ldots m$. Note $\eta$ consists of $\sum_{j=1}^{l} t_{j}$ independent chi-squared $\eta_{j, k}$ with $2 m$ degrees of freedom, whose characteristic function (CF) $\Phi_{\eta_{j, k}}(s)$ is given as $\Phi_{\eta_{j, k}}(s)=\int_{0}^{\infty} f_{\eta_{j, k}}(x) e^{i s x} d x=$ $\left(1-s i a_{j} \lambda_{j, k} w_{j} \rho / q_{j}\right)^{-m}$, where $f_{\eta_{j, k}}(x)$ denotes the probability density function (PDF) of $\eta_{j, k}$. Assuming each $a_{j} \lambda_{j, k} w_{j} \rho / q_{j}$ differs, the CF of $\eta$ can then be obtained and resolved into its partial fractions

$$
\begin{aligned}
\Phi_{\eta}(s) & =\prod_{j=1}^{l} \prod_{k=1}^{t_{j}} \Phi_{\eta_{j, k}}(s) \\
& =\sum_{j=1}^{l} \sum_{k=1}^{t_{j}} \sum_{d=1}^{m} q_{j, k, d}\left(1-s i a_{j} \lambda_{j, k} w_{j} \rho / q_{j}\right)^{-d}
\end{aligned}
$$

where $q_{j, k, d}$ are derived by solving a system of linear equations

$$
\begin{aligned}
& q_{j, k, d}=\frac{\left(-i a_{j} \lambda_{j, k} w_{j} \rho / q_{j}\right)^{-(m-d)}}{(m-d) !} \frac{\partial^{m-d}}{\partial s^{m-d}} \\
& \times\left(\prod_{u=1, u \neq j}^{l} \prod_{v=1, v \neq k}^{t_{j}}\left(1-s i a_{u} \lambda_{u, v} w_{u} \rho / q_{u}\right)^{-m}\right)_{s=q_{j} / i a_{j} \lambda_{j, k} w_{j} \rho}
\end{aligned}
$$

The PDF of $\eta$ can therefore be obtained by inverse transformation to $\Phi_{\eta}(s)$

$$
f_{\eta}(x)=\sum_{j=1}^{l} \sum_{k=1}^{t_{j}} \sum_{d=1}^{m} \frac{q_{j, k, d}}{\Gamma(d)}\left(\frac{a_{j} \lambda_{j, k} w_{j} \rho}{q_{j}}\right)^{-d} x^{d-1} e^{-\frac{x q_{j}}{a_{j} \lambda_{j, k} w_{j} \rho}}
$$

with Gamma function $\Gamma(x)$. Finally, the outage probability $P_{o}$ with a data rate $R$ can be calculated

$$
\begin{aligned}
P_{o} & =P(C<R)=P\left(\eta<2^{R / r_{l}}-1\right)=\int_{0}^{2^{R / r_{l}}-1} f_{\eta}(x) d x \\
& =\sum_{j=1}^{l} \sum_{k=1}^{t_{j}} \sum_{d=1}^{m} \frac{q_{j, k, d}}{\Gamma(d)} \Gamma\left(d, \frac{\left(2^{R / r_{l}}-1\right) q_{j}}{a_{j} \lambda_{j, k} w_{j} \rho}\right)
\end{aligned}
$$

where $\Gamma(a, x)=\int_{0}^{x} y^{a-1} e^{-y} d y$ is the lower incomplete Gamma function. For OSTBCs transmitted over any other subset, the outage probability can also be obtained. We observe that only with the eigenvalues of $\mathbf{R}_{t}$ at the transmitter, we can minimize the outage probability of each subset and then select the subset with the best outage performance for transmission. Since directly optimizing the outage probability is difficult, we resort to the following suboptimal scheme.

\section{Antenna Subset Selection With Suboptimal POWER ALlocATION SCHEME}

At different TSNR levels, the optimal transmission of OSTBCs may employ different subsets. Intuitively, at high TSNR levels, subset $\mathrm{A}_{l}$ is optimal due to its high diversity order, while at some lower TSNR levels, fewer ports may perform better 
because DAs with severe large-scale fading may waste power. At TSNR level $\rho$, we assume subset $\mathrm{A}_{l}$ with positive weights $w_{1}, \ldots, w_{l}$ is optimal. The optimal outage probability $P_{o, \mathrm{~A}_{l}}^{o p t}$ can be written in terms of $\Phi_{\eta}(s)$

$$
\begin{aligned}
& P_{o, A_{l}}^{o p t}= \int_{0}^{2^{R / r_{l}}-1} f_{\eta}(x) d x \\
&= \int_{0}^{2^{R / r_{l}}-1}\left(\int_{-\infty}^{+\infty} \Phi_{\eta}(s) e^{-i s x} d s\right) d x \\
&= \int_{0}^{2^{R / r_{l}}} \int_{-\infty}^{-1} \prod_{j=1}^{+\infty} \prod_{k=1}^{t_{j}}\left(\left(1-\frac{s i a_{j} w_{j} \rho \lambda_{j, k}}{q_{j}}\right)^{-m \sum_{j=1}^{l} t_{j}}\right. \\
&\left.\times e^{-i s x}\right)^{\frac{1}{\sum_{j=1}^{l} t_{j}}} d s d x
\end{aligned}
$$

Since $\mathrm{A}_{l}$ is assumed optimal, the contribution of each DA port is similar. Denote $m \sum_{j=1}^{l} t_{j}$ by $D, P_{o, \mathrm{~A}_{l}}^{o p t}$ can be approximated by

$$
\begin{aligned}
& P_{o, A_{l}}^{o p t} \\
& \approx \int_{0}^{2^{R / r_{l}}-1} \prod_{j=1}^{l} \prod_{k=1}^{t_{j}}\left(\int_{-\infty}^{+\infty}\left(1-\frac{s i a_{j} \lambda_{j, k} w_{j} \rho}{q_{j}}\right)^{-D}\right. \\
& \left.\times e^{-i s x} d s\right)^{\frac{m}{D}} d x \\
& =\int_{0}^{2^{R / r_{l}}-1} \frac{x^{D-1}}{\Gamma(D)} \prod_{j=1}^{l} \prod_{k=1}^{t_{j}}\left(\left(\frac{a_{j} \lambda_{j, k} w_{j} \rho}{q_{j}}\right)^{-m}\right. \\
& \left.\times e^{-\frac{q_{j} x}{a_{j} \lambda_{j, k^{2} w_{j} \rho}} \frac{m}{D}}\right) d x=P_{o, A_{l}}^{\text {Approx. }} .
\end{aligned}
$$

Note $e^{-x} \leq 1$ for $x \geq 0$, the upper bound of $P_{o, A_{l}}^{\text {Approx. }}$ is derived to be

$$
\begin{aligned}
& P_{o, A_{l}}^{\text {Approx. }} \leq\left(\frac{2^{R / r_{l}}-1}{\rho}\right)^{D} \frac{D^{-1}}{\Gamma(D)} \\
& \quad \times \prod_{j=1}^{l} \prod_{k=1}^{t_{j}}\left(\frac{a_{j} w_{j} \lambda_{j, k}}{q_{j}}\right)^{-m}=P_{o, A_{l}}^{u b} .
\end{aligned}
$$

The suboptimal power allocation vector $\mathbf{w}^{*}=\left[w_{1}^{*}, \ldots, w_{l}^{*}\right]$ can be obtained by minimizing $P_{o, A_{l}}^{u b}$, thus reducing to the following optimization problem:

$$
w^{*}=\arg \max \prod_{j=1}^{l}\left(w_{j}\right)^{t_{j}}, \quad \text { subject to } \sum_{j=1}^{l} w_{j}=1 .
$$

Using the Lagrangian method, we obtain $w_{j}^{*}$ as

$$
w_{j}^{*}=t_{j} / \sum_{j=1}^{l} t_{j}, j=1, \ldots, l .
$$

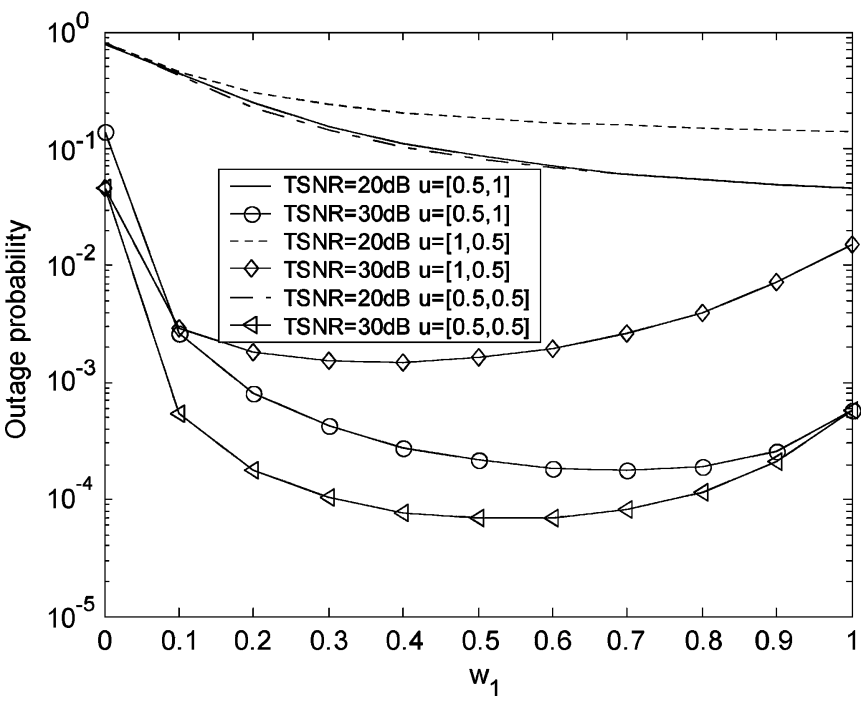

Fig. 2. Outage probability versus $w_{1}$ (power allocation weight to Port 1 ) of $3 / 4$ rate OSTBCs.

Note that the above power allocation scheme is accurate in the high TSNR region because $P_{o, \mathrm{~A}_{l}}^{u b}$ is tight when $\rho$ is high enough. Substituting (12) into (7), the suboptimal outage probability $P_{o, A_{l}}^{s u b}$ can be calculated. Employing the above method to any optimal antenna subset, a similar suboptimal power allocation scheme can be easily derived, i.e., to allocate transmit power proportional to the rank of the transmit correlation matrix of each port. To obtain the optimal subset, we apply the suboptimal power allocation scheme to any possible optimal subset $\mathrm{A}_{k}(k=$ $\left.1, \ldots, 2^{l}-1\right)$ to obtain the corresponding suboptimal outage probability $P_{o, A_{k}}^{s u b}$. The subset with the best outage performance shall be selected. Consequently, the suboptimal outage probability of OSTBCs transmitted over the $\left(n, l, q_{1}, \ldots, q_{l}, m\right)$ DA topology, $P_{o}^{s u b}$, is obtained as

$$
P_{o}^{s u b}=\min \left(P_{o, A_{1}}^{s u b}, P_{o, A_{2}}^{s u b}, \ldots, P_{o, A_{2} l_{-1}}^{s u b}\right) .
$$

The above power allocation results can also be obtained at the receiver and fed back to the transmitter. However, complexity of receiver is increased, and we do not discuss this case here.

\section{NUMERICAL RESULTS}

Consider a $(4,2,2,2,1)$ DA topology where 4 four DAs are grouped into two ports and the receiver has one antenna, with the normalized large-scale fading parameters $a_{1}=1$ and $a_{2}=0.1$. The targeted capacity is $3 \mathrm{bits} / \mathrm{s} / \mathrm{Hz}$. The antenna subsets include $\mathrm{A}_{1}$ (Port $1 \mathrm{DAs}$ ), $\mathrm{A}_{2}$ (2 ports DAs), and $\mathrm{A}_{3}$ (Port $2 \mathrm{DAs}$ ). The $3 / 4$ rate OSTBCs are transmitted on $A_{2}$, and full rate OSTBCs are transmitted on $A_{1}$ and $A_{3}$. The transmit correlation coefficients are denoted by $\mathbf{u}=\left[u_{1}, u_{2}\right]$, where $u_{1}$ and $u_{2}$ denote the correlation coefficient between the two DAs of Ports 1 and 2, respectively. $\mathbf{R}_{t, 1}$ and $\mathbf{R}_{t, 2}$ are then given as $\mathbf{R}_{t, 1}=\left[1 u_{1} ; u_{1} 1\right]$ and $\mathbf{R}_{t, 2}=\left[\begin{array}{ll}1 & u_{2} ; u_{2} \\ 1\end{array}\right]$.

For 3/4 rate OSTBCs over subset $\mathrm{A}_{2}$, Fig. 2 depicts the outage probability versus power allocation weight to Port $1\left(w_{1}\right)$ in three correlation scenarios, i.e., $\mathbf{u}=[0.5,0.5], \mathbf{u}=[0.5,1]$, 


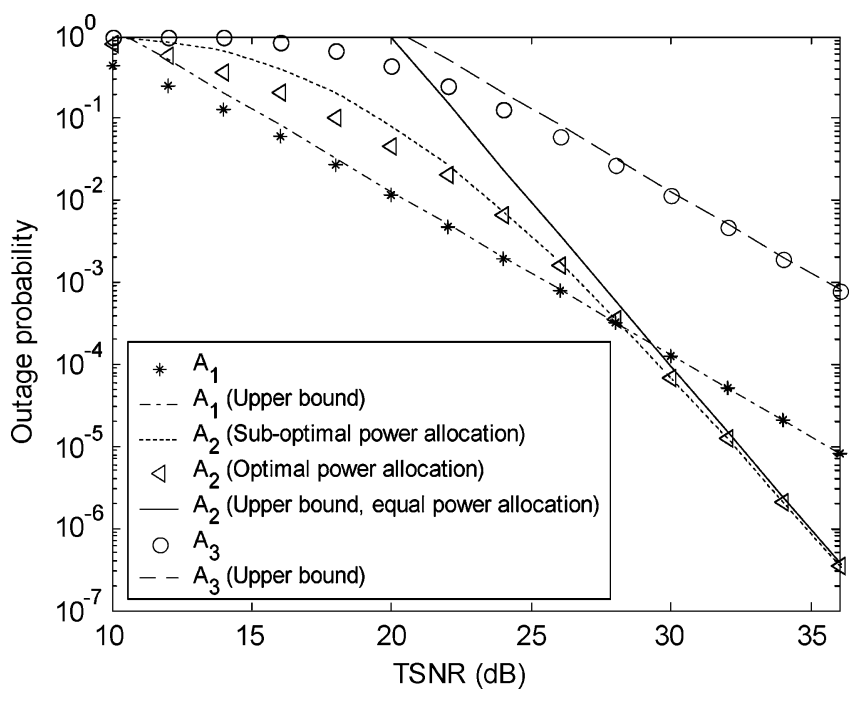

Fig. 3. Outage probability versus TSNR of OSTBCs over different subsets.

and $\mathbf{u}=[1,0.5]$. When TSNR is $20 \mathrm{~dB}$, the outage curves decrease monotonously as $w_{1}$ increases, indicating the optimality of transmission over $\mathrm{A}_{1}$. However, at TSNR level of $30 \mathrm{~dB}, \mathrm{~A}_{2}$ is optimal, with the optimal $w_{1}$ being about $1 / 2,2 / 3$, and $1 / 3$ for the three scenarios, respectively. These results verify that the suboptimal power allocation scheme is very close to optimal. For example, according to (12), when $\mathrm{A}_{1}$ is optimal, the suboptimal $w_{1}^{*}$ is 1 , which is exactly the optimal value, and when $\mathrm{A}_{2}$ is optimal, the suboptimal $w_{1}^{*}$ is $1 / 2,2 / 3$, and $1 / 3$ for the three scenarios, which are very close to optimal.

Antenna subset selection with suboptimal power allocation scheme is also illustrated by the above system in the scenario where $\mathbf{u}=[0.5,0.5]$. Fig. 3 shows the outage probability of $\mathrm{A}_{1}, \mathrm{~A}_{3}, \mathrm{~A}_{2}$ with suboptimal power allocation (i.e., equal power allocation in this case) and $\mathrm{A}_{2}$ with optimal power allocation obtained from numerical optimization. The upper bounds on the outage probability approximations of $\mathrm{A}_{1}, \mathrm{~A}_{3}$, and $\mathrm{A}_{2}$ with equal power allocation are also depicted. We observe that these upper bounds are tight in high TSNR regions. Also, we find the suboptimal performance of $\mathrm{A}_{2}$ is very close to the optimal performance at high TSNR levels. Subset selection is easy but effective, e.g., $A_{1}$ is selected when TSNR is below $28 \mathrm{~dB}$, and $\mathrm{A}_{2}$ is selected when TSNR is beyond $28 \mathrm{~dB}$. With subset selection, the suboptimal performance in (13) is very close to optimal at any TSNR level. The benefit of subset selection compared with equal power transmission over $\mathrm{A}_{2}$ is obvious, e.g., a 3-dB reduction in TSNR at a $10^{-2}$ outage level. Finally, we gladly observe that subset selection based on the upper bounds of outage probability approximation yields almost the same result but with significantly reduced complexity.

\section{REFERENCES}

[1] W. Roh and A. Paulraj, "MIMO channel capacity for the distributed antenna systems," in Proc. IEEE VTC, 2002, vol. 2, pp. 706-709.

[2] E. G. Larsson and P. Stoica, Space Time Block Coding for Wireless Communications. Cambridge, U.K.: Cambridge Univ. Press, 2003.

[3] H. Zhuang, L. Dai, L. Xiao, and Y. Yao, "Spectral efficiency of distributed antenna system with random antenna layout," Electron. Lett., vol. 39, no. 6, pp. 495-496, 2003.

[4] S. Zhou, M. Zhao, X. Xu, J. Wang, and Y. Yao, "Distributed wireless communication system: a new architecture for future public wireless access," IEEE Commun. Mag., vol. 41, no. 3, pp. 108-113, Mar. 2003.

[5] V. Tarokh, H. Jafarkhani, and A. R. Calderbank, "Space-time block codes from orthogonal designs," IEEE Trans. Inf. Theory, vol. 45, no. 7, pp. 1456-1467, Jul. 1999.

[6] C. N. Chuah, D. N. C. Tse, and J. M. Kahn, "Capacity scaling in MIMO wireless systems under correlated fading," IEEE Trans. Inf. Theory, vol. 48, no. 3, pp. 637-650, Mar. 2002.

[7] S. Han, S. Zhou, J. Wang, and W. Park, "Transmit antenna selection with power and rate allocation for spatial multiplexing in distributed antenna systems," Tsinghua Sci. Technol., vol. 11, no. 3, pp. 259-263, Jun. 2006.

[8] M. Dohler and H. Aghvami, "Information outage probability of distributed STBCs over Nakagami fading channels," IEEE Commun. Lett., vol. 8, no. 7, pp. 437-439, Jul. 2004. 